\title{
BIOPHOTONICS
}

\section{That quantum feeling}

By engineering photosensitive proteins and tweaking the classical properties of light, it should be possible to tune the response of a cell equipped with photoreceptors. But would these cells be able to sense the subtle quantum properties of light?

\section{Elisabetta Collini}

$\mathrm{D}$ ebate has long raged over whether biological functions follow the principles of quantum mechanics ${ }^{1,2}$. Light harvesting ${ }^{3}$ and olfaction ${ }^{4}$ are among the most cited systems in this discussion, but it has even been suggested that quantum mechanics may be a biasing force in evolution ${ }^{5}$. And other sensory mechanisms, such as vision ${ }^{6}$ and neuronal communications ${ }^{7}$, are quickly gaining ground. One of the main sources of opposition to these ideas is the difficulty in correlating the possible presence of quantum mechanisms in vitro and the effective biological behaviour in vivo. Now, writing in Nature Physics, Kush Paul and co-workers ${ }^{8}$ have established a direct correlation between an optical stimulus and the control of biological functions, by studying the response of a photoreceptor known as channelrhodopsin-2 (ChR2) in living brain tissue.

In living organisms, sensory information is processed by specific receptors that are capable of translating an external stimulus into an electrical potential, which then travels to a specific region of the brain to be interpreted. Opsins fall into a particular class of receptor known as photoreceptors, which are activated by external luminous stimuli. These light-gated ion channels are able to transduce a light signal into an electrical response, or an ionic current, which controls neuronal activity (Fig. 1a).

By directly measuring the current output after stimulation with light, Paul et al. demonstrated that the amount of neuronal activity associated with ChR2 strongly depends on the coherent properties of the light pulse used as stimulus (Fig. 1b,c). To achieve this result, they applied the strategies of quantum control, a method for controlling dynamical processes by light. The basic principle is to control quantum phenomena - typically by modulating the phase and the chirp of a laser pulse? Previous experiments had succeeded in verifying that it is possible to manipulate the chemical reaction at the base of opsin activity using tailored quantum coherent controlled pulses, but they were unable

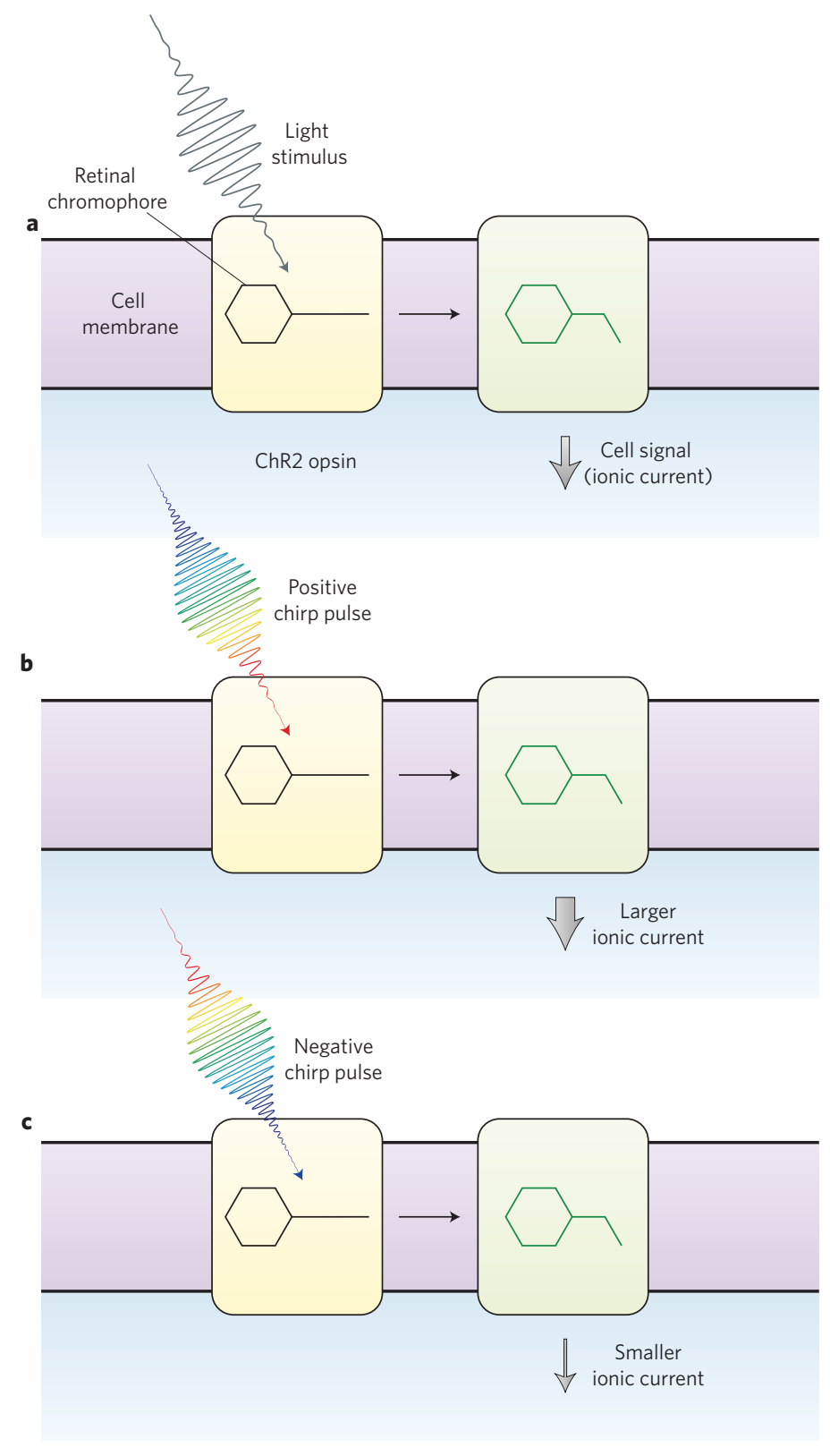

Figure 1 | Light transduction by opsin. a, The absorption of a photon by the retinal chromophore induces a conformational change that leads to the opening of a pore, through which ions can flow to generate an ionic current. $\mathbf{b}$, Stimulation with positively chirped pulses, phase shaped so that the low-frequency components are in the leading edge, invokes a larger ionic current. c, Negatively chirped pulses (with high frequencies in the leading edge) result in a smaller ionic current. 
to draw a clear correlation between spectroscopic observables and opsin activity in a living cell ${ }^{10,11}$.

The results of Paul et al. are a first step in this direction and provide us with a somewhat different point of view: namely, that the question of whether nature exploits quantum mechanics should probably be replaced by whether nature is capable of responding to quantum mechanics. If the answer is yes, as now seems plausible, then the design of suitable tailored pulses may enable us to directly control cellular activities.

One could legitimately object that the photo-triggering light stimuli affecting real living systems are not phase-shaped laser pulses. This is certainly a sound argument. But producing suitably tailored quantum coherent light is becoming increasingly easier these days. And there is mounting evidence suggesting that biological complexes, such as opsins and antenna complexes, seem to be able to respond to quantum stimuli. This implies that - even if nature does not function quantum mechanically in biologically relevant conditions - it may still be possible to steer and control biological activity by manipulating quantum effects. In other words, quantum coherent control of living matter could be achieved. This is an unexpected - but very appealing development that can be framed in the wider context of quantum technologies.

Elisabetta Collini is in the Department of Chemical Sciences at the University of Padova,
35131 Padova, Italy.

e-mail:elisabetta.collini@unipd.it

References

1. Huelga, S. F. \& Plenio, M. B. Contemp. Phys. 54, 181-207 (2013).

2. Scholes, G. D. et al. Nature 543, 647-656 (2017).

3. Chenu, A. \& Scholes, G. D. Annu. Rev. Phys. Chem. 66, 69-96 (2015).

4. Bittner, E. R., Madalan, A., Czader, A. \& Roman, G. J. Chem. Phys. 137, 22A551 (2012)

5. McFadden, J. Quantum Evolution (HarperCollins, 2000).

6. Johnson, P. J. M. et al. Nat. Chem. 7, 980-986 (2015).

7. Deng, D.-L, Li, X. \& Das Sarma, S. Phys. Rev. X 7, $021021(2017)$

8. Paul, K. et al. Nat. Phys. 13, 1111-1116 (2017).

9. Shapiro, M. \& Brumer, P. Adv. At. Mol. Opt. Phys. 42, 287-345 (2000)

10. Prokhorenko, V. I. et al. Science

313, 1257-1261 (2006)

11. Polli, D. et al. Nature 467, 440-443 (2010).

Published online: 18 September 2017

\section{Kitaev physics crystalized}

A state of matter known as a quantum spin liquid has been predicted to host Majorana fermions. Recent neutron scattering and specific heat results add to the growing body of evidence suggesting they exist in the quantum magnet $\alpha-\mathrm{RuCl}_{3}$.

\section{Natalia Perkins}

$\mathrm{M}$ agnetic insulators are usually associated with long-range magnetic order mediated by the exchange interaction between localized spins. However, in certain circumstances this may not be the case, and a peculiar state displaying no long-range order down to zero temperature can arise, even if the spins are strongly interacting. This state is called a quantum spin liquid (QSL) $)^{1,2}$, of which the Kitaev spin liquid on the honeycomb lattice ${ }^{3}$ is a quintessential example.

The QSL state of matter is very difficult to detect experimentally because it lacks a local order parameter to which common probes couple directly. The three defining characteristics of a QSL are a topological ground-state degeneracy, long-range entanglement, and a fractionalization of the fundamental spin degrees of freedom. While the first two are not easily accessible by experiment, the last one can potentially be detected by means of thermodynamic and spin structure factor measurements.

Although a range of material systems have been proposed as experimental realizations of the Kitaev model, a flurry of recent experiments focusing on $\alpha-\mathrm{RuCl}_{3}$ suggest this system is particularly promising ${ }^{4-6}$. Now, writing in Nature Physics, Sungdae Ji and collaborators ${ }^{7}$ claim to have observed the signature of spin fractionalization in high-quality a- $\mathrm{RuCl}_{3}$ crystals.

The combination of specific heat measurements and inelastic neutron scattering data presented by the authors reveals unusual magnetic excitations: low-energy quasi-elastic excitations around the Brillouin zone centre and an hour-glass-like magnetic continuum at high energies. These findings are in agreement with a recent inelastic neutron scattering study by Banerjee et al. ${ }^{6}$, who also suggested that temperature, energy and momentum dependences of magnetic excitation in $\alpha-\mathrm{RuCl}_{3}$ are consistent with the Kitaev model ${ }^{3}$.

Ji and colleagues argue that, at temperatures above $6.5 \mathrm{~K}$ (below which $\mathrm{RuCl}_{3}$ develops a long-range magnetic order), the spins fractionalize into itinerant fermions and localized magnetic fluxes, both of which are composed of Majorana fermions. This claim is supported by a careful data analysis of the specific heat and inelastic neutron scattering experiments, and comparison of the experimental findings with the results of the cluster dynamical mean-field theory and continuous-time quantum Monte Carlo simulations ${ }^{8}$.

In particular, the authors observe a rather peculiar behaviour in the magnetic specific heat: above the ordering temperature it exhibits two broad maxima - one around $50 \mathrm{~K}$ and the other around $100 \mathrm{~K}$ - with a linear temperature dependence between them, indicating a metallic-like nature of excitations. This is consistent with the expected properties of quasiparticles emerging from the fractionalization of the Kitaev QSL 9 .

The authors suggest the following interpretation of the observed finitetemperature phase diagram (Fig. 1): the two characteristic crossovers observed at finite temperatures correspond to a two-stage release of magnetic entropy, first through itinerant fermions and then through localized Majorana fermions. In other words, they argue that below $50 \mathrm{~K}$, magnetic fluxes are frozen in the topologically ordered 